\title{
Study of Pluto's atmosphere based on 2020 stellar occultation light curve results
}

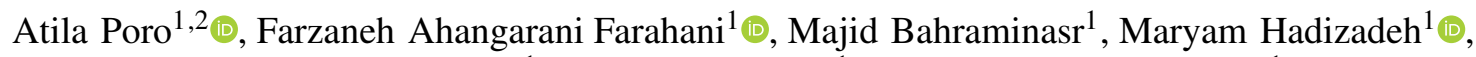 \\ Fatemeh Najafi Kodini ${ }^{1}{ }^{(D}$, Maryam Rezaee $^{1}{ }^{(D}$, and Mahsa Seifi Gargari ${ }^{1}{ }^{(D}$ \\ 1 The International Occultation Timing Association Middle East Section, Iran \\ e-mail: info@iota-me.com \\ 2 Astronomy Department of the Raderon Lab., Burnaby, BC, Canada \\ Received 6 July 2021 / Accepted 2 September 2021
}

\section{ABSTRACT}

\begin{abstract}
On 6 June 2020, Pluto's stellar occultation was successfully observed at a ground-based observatory in Iran, and Pluto's atmospheric parameters were investigated. We used an atmospheric model of Pluto, assuming a spherical and transparent pure $\mathrm{N}_{2}$ atmosphere. Using ray-tracing code, the stellar occultation light curve was satisfactorily fit to this model. We found that Pluto's atmospheric pressure at the reference radius of $1215 \mathrm{~km}$ was $6.72 \pm 0.48 \mu \mathrm{bar}$ in June 2020 . Our estimated pressure shows a continuation of the pressure increase trend observed since 1988 and does not confirm the rapid pressure decrease tentatively reported in 2019 . The pressure evolution is consistent with a seasonal transport model. We conclude that the $\mathrm{N}_{2}$ sublimation process from Sputnik Planitia is continuing. This study's result is shown on the diagram of the annual evolution of atmospheric pressure.
\end{abstract}

Key words. occultations - planets and satellites: atmospheres - Kuiper belt objects: individual: Pluto

\section{Introduction}

Pluto and its satellites are the best and most comprehensible of the trans-Neptunian objects (TNOs) due to their well-known complicated and active geological properties (Spencer et al. 2020). The Hubble Space Telescope observation revealed some features of this dwarf planet. Furthermore, NASA's New Horizons spacecraft made a close flyby of Pluto in 2015 in order to study Pluto and its moon Charon (Stern et al. 2015). Since the confirmation of the existence of Pluto's atmosphere based on a 1988 stellar occultation (Hubbard et al. 1988), the study of Pluto's atmospheric parameters (pressure, composition, temperature, etc.) has carried on using data from both ground-based and space-based observation.

To calculate Pluto's atmospheric pressure, Elliot \& Young (1992) developed a model based on a thermal gradient indicated in a light curve as a scale height. This model is a method for precisely coordinating the data obtained from an occultation light curve. The structure of Pluto's atmosphere was determined in the areas examined by the occultation. Additionally, the conceivable physical conditions of the atmosphere on the supposition were investigated (Elliot et al. 1989). Later, in 2015, a simple atmospheric model, named DO15, was defined based on the use of both direct ray-tracing and inversion approaches by assuming a spherically symmetric, clear, and pure $\mathrm{N}_{2}$ atmosphere (Dias-Oliveira et al. 2015). This model was suitably fit with light curves from 2012 and 2013 stellar occultations between the heights of $1190 \mathrm{~km}$ and $1450 \mathrm{~km}$ from Pluto's center (Dias-Oliveira et al. 2015).

Research conducted in recent decades has indicated that Pluto's atmospheric pressure changes are due to the seasonal cycles of Pluto's surface volatiles, which were calculated using atmospheric models (Meza et al. 2019). In 1988, atmospheric pressure at a radius of $1215 \mathrm{~km}$ (the distance to Pluto's center) was estimated at $2.33 \pm 0.24 \mu$ bar (Yelle \& Elliot 1997). Atmospheric pressure increased to $6.05 \pm 0.32 \mu \mathrm{bar}$ in 2008 (Sicardy et al. 2011). A drop in Pluto's atmospheric pressure was reported from 2008 to 2010 , estimated at $5.64 \pm 0.22 \mu$ bar (Young et al. 2010). The Stratospheric Observatory for Infrared Astronomy (SOFIA) aircraft, simultaneously with ground-based observatories, observed the 2015 stellar occultation by Pluto at an altitude of $\sim 40000 \mathrm{ft}$. (Bosh et al. 2015). Analysis of the data from SOFIA in optical and near-infrared wavelengths, along with ground-based observations, showed that the atmospheric pressure at half-light altitude was stable from 2011 to 2015 (Bosh et al. 2015). In 2015, Pluto's atmospheric pressure was observed at its maximum value of about $6.92 \pm 0.07 \mu$ bar (Sicardy et al. 2016). According to a stellar occultation observation in 2019, Pluto's atmospheric pressure was estimated to be $5.20_{-0.19}^{+0.28} \mu$ bar, which showed a decrease of approximately $21 \%$ between 2016 and 2019 at the $2.4 \sigma$ level (Arimatsu et al. 2020).

In this study we observed a stellar occultation by Pluto on 6 June 2020. These observational data were used to obtain the atmospheric pressure of Pluto. The result and discussion about the obtained parameters are presented.

\section{Observation and data reduction}

The occultation of the star Gaia DR2 ID: 6864932072159710592 , with an astrometric position of RA: $19^{\mathrm{h}} 45^{\mathrm{m}} 33.8957^{\mathrm{s}}$ Dec: $-22^{\circ} 10^{\mathrm{m}} 19.0471^{\mathrm{s}} \quad(\mathrm{J} 2000)$ and $G$ magnitude of $12.97871^{1}$, occurred on 6 June 2020 (UT).

1 http://vizier.u-strasbg.fr/ 
According to the prediction by the Lucky Star project ${ }^{2}$, the observation was made with the equipment of a private Asal observatory in the north of Karaj City, Iran. To improve observing circumstances, a place was identified at a height not far from the observatory, and the observatory's equipment was transferred there. The observation was carried out at the geographical coordinates $35^{\circ} 51^{\prime} 48^{\prime \prime} \mathrm{N}$ and $51^{\circ} 01^{\prime} 20^{\prime \prime} \mathrm{E}$ and 2220 meters above the mean sea level, and the horizon was free of any obstructions. This event was recorded with a 24-inch Schmidt-Cassegrain as the main telescope and a 14-inch Schmidt-Cassegrain telescope installed on the main telescope; both telescopes were on a Paramount equatorial mount. The observation was undertaken using a clear filter. Photometric data were derived from two CCD. SBIG 11000M cameras with a $2004 \times 1336$ pixel array and pixel length of $18 \mu$ on the both telescopes. During the occultation the exposure time to cycle ratio was 0.81 seconds (dead time $=0.93 \mathrm{~s}$ ), and the average temperature of the $\mathrm{CCD}$ was $-25^{\circ} \mathrm{C}$. The CCDs took images at exactly the same time under the same conditions. All the simultaneous images were merged into pairs to get an integrated image. The raw images were aligned by AstroImageJ (AIJ) software (Collins et al. 2017). DeepSkyStacker software (Ashley 2015) was used to merge each pair of simultaneous images. This image processing was necessary based on the low altitude of the event, the exposure time for each telescope's image, and a large amount of light reduction of $\sim 1.9$ magnitude. A total of 112 images were taken, of which 25 were usable images taken at the time of the occultation. We only used images that were in good condition on both telescopes at the same time. It should be noted that some of the images have been deleted due to high noise or sudden atmospheric turbulence. The Pluto elevation at the beginning and end of the observation was $\sim 5$ and $\sim 9$ degrees, respectively.

We reduced the images and corrected them. The data reduction was performed for bias, dark, and flat-field of each CCD image according to the standard method based on AIJ software. As light passes through the Earth's atmosphere, the airmass decreases the flux of stars. We estimated the airmass based on observatory coordinates and the position of the star in the sky during the observation using Hardie's formula (Hiltner 1962). This was accomplished using a Python code based on the Astropy package (Astropy Collaboration 2013) and then applied to the light curve utilizing AIJ. Applying airmass improved the light curve for the entire length of our observation. The flux was then normalized to the out-of-occultation profile.

Figure 1 shows the Earth as seen from Pluto at the time of the occultation. The outer thin blue lines correspond to a predicted $1 \%$ drop in light from the star, the practical limit of detection, and the inner thin blue lines correspond to the zone where a halflight level was to be expected.

\section{Method of analysis and light curve fitting}

A best fit of an atmospheric model was done to the occultation light curve data set. The extracted light curve is the total flux from the star and Pluto's system as a function of time (in JD). The light curve after normalization is shown in Fig. 2.

The atmospheric pressure of Pluto is a time-dependent parameter that has changed over time. Since the main component of Pluto's atmosphere is nitrogen $\left(\mathrm{N}_{2}\right)$, atmospheric pressure deviations have been caused by continuous $\mathrm{N}_{2}$ sublimation/condensation processes in the Sputnik Planitia glacier

\footnotetext{
2 https://lesia.obspm.fr/lucky-star/index.php
}

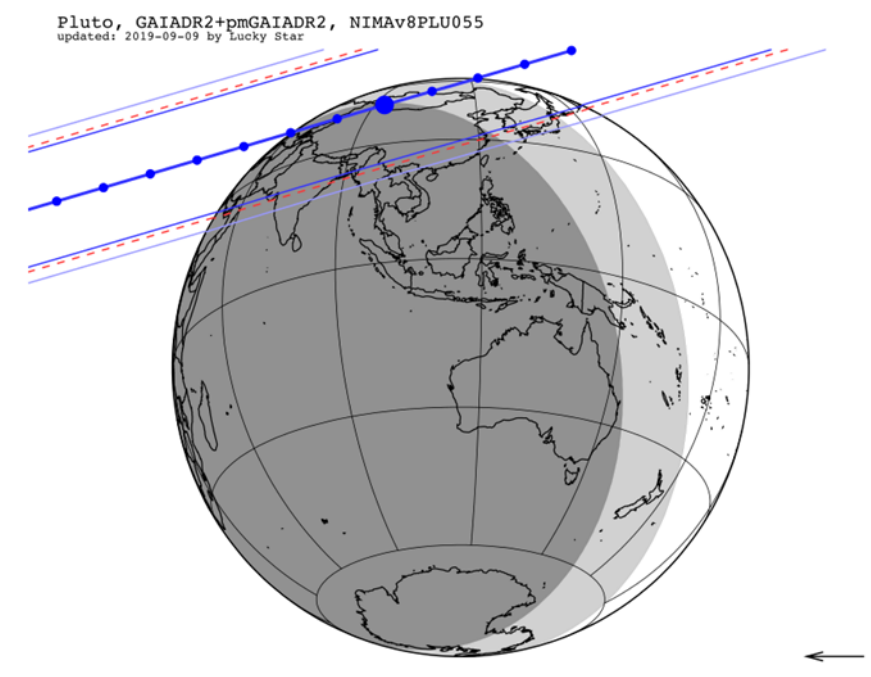

Fig. 1. Path of Pluto's shadow on Earth on 6 June 2020. Small blue dots show the track of central occultation geographically each minute before and after the predicted time of central occultation. The large dot indicates the location/time of central occultation in China. The arrow corresponds to the direction of the shadow motion. The precision of the dashed red line is the estimated $1 \sigma$ path deviation. When the Sun elevation is below -18 degrees (night) the areas are dark gray, and when the Sun elevation is between 0 and -18 degrees areas are shown in light gray. The prediction comes from the Lucky Star project.

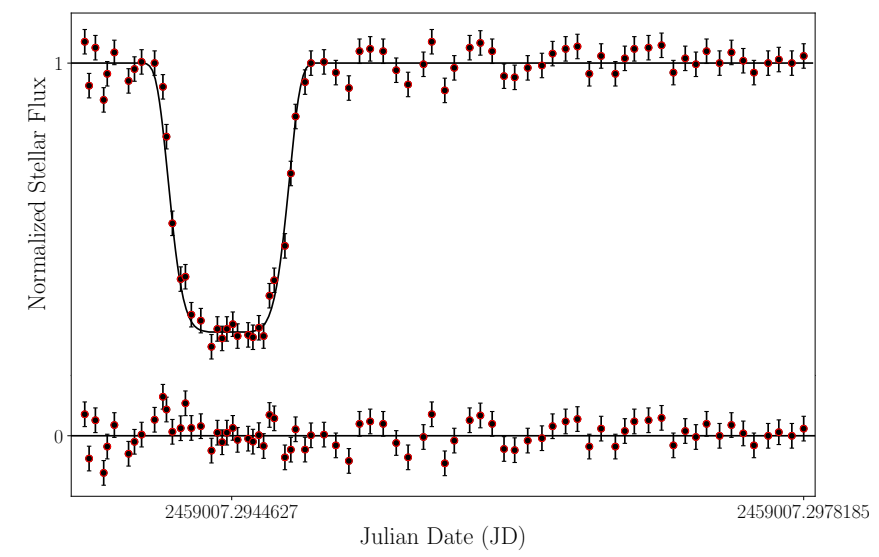

Fig. 2. Normalized occultation light curve with the best-fit atmospheric model from the 6 June 2020 occultation. The solid curve shows a simultaneous fit to our light curve using the DO15 atmospheric model. The residuals are also added at the bottom of the panel. The light curve shows part of the data that was out of occultation.

(Stern et al. 2015; Sicardy et al. 2016). In order to obtain the pressure of Pluto's atmosphere, we fit the occultation light curve through a spherical and transparent atmospheric model of Pluto with a synthetic profile (Dias-Oliveira et al. 2015). This was created by using our ray-tracing code based on the pressure at the reference radius of $R=1215 \mathrm{~km}$ (Sicardy et al. 2016; Meza et al. 2019; Arimatsu et al. 2020).

This event was observed in low-elevation conditions with a clear filter and airmass changes. We used the Pluto+Charon flux as an additional free parameter in our analysis process, constant with time (i.e., we assumed that the (Pluto+Charon)/star flux ratio was independent of airmass). Table 1 shows the constant physical parameters that are used in this technique. We used the same parameters as in Dias-Oliveira et al. (2015) and Meza et al. (2019). 
Table 1. Physical constant and adopted parameters for the 6 June 2020 light curve fit and results.

\begin{tabular}{lc}
\hline \hline Parameter & Value \\
\hline Pluto's mass $\left(G M_{\mathrm{p}}\right)$ & $8.69 \times 10^{11} \mathrm{~m}^{3} \mathrm{~s}^{-2}$ \\
Surface radius $\left(R_{\text {surface }}\right)$ & $1187 \mathrm{~km}$ \\
Pluto's geocentric distance $(\mathrm{D})$ & $4.9889 \times 10^{9} \mathrm{~km}$ \\
Pluto pole position $(\mathrm{J} 2000)$ & $\alpha_{\mathrm{p}}=08^{\mathrm{h}} 52^{\mathrm{m}} 12.94^{\mathrm{s}}, \delta_{\mathrm{p}}=-06^{\mathrm{d}} 10^{\prime} 04.8^{\prime \prime}$ \\
$\mathrm{N}_{2}$ molecular refractivity $(\mathrm{K})$ & $1.091 \times 10^{-23}$ \\
\hline Pressure at 1215 km $\left(P_{1215}\right)$ & $6.72 \pm 0.48 \mu \mathrm{bar}$ \\
Pressure at the surface $\left(P_{\text {surface }}\right)$ & $12.36 \pm 0.38 \mu \mathrm{bar}$ \\
Closest approach to Pluto's shadow center & $605.3 \pm 5 \mathrm{~km}$ \\
Time of closest approach to shadow center $(\mathrm{UT})$ & $19^{\mathrm{h}} 4^{\mathrm{m}} 1.58 \pm 0.56^{\mathrm{s}}$ on $2020 \mathrm{June} 06$ \\
(Pluto+Charon)/star flux ratio & $0.313 \pm 0.054$ \\
\hline
\end{tabular}

As in the DO15 atmospheric model described by Dias-Oliveira et al. (2015), all the equations depend only on the radius distance from Pluto's center. By assuming a spherically symmetric planet and an $\mathrm{N}_{2}$ pure atmosphere in the DO15 model, we satisfactorily fit our light curve. To demonstrate the quality of fit, the $\chi^{2}$ per degree of freedom was obtained by a well-known relation,

$\chi_{\text {d.o.f. }}^{2}=\frac{\chi^{2}}{N-M}=\frac{1}{N-M} \sum_{i=1}^{N} \frac{\left(\Phi_{i, \mathrm{obs}}-\Phi_{i, \mathrm{syn}}\right)^{2}}{\left(\sigma_{i}\right)^{2}}$,

where: for the $i$ th data point, $\Phi_{i, \mathrm{obs}}$ and $\Phi_{i, \mathrm{syn}}$ are the observed and synthetic stellar fluxes, respectively, with $\sigma$ error for each data point; $N$ is all the light curve data points; and $M$ is the number of free parameters according to the model. The $\chi^{2}$ map as a function of the surface pressure, $P_{\text {surface, }}$, and the distance of the closest approach of our observatory to Pluto's shadow center, $\rho$, is shown in Fig. 3. According to the simultaneous fit to our occultation light curve, the $\chi^{2}$ value is 10.08 with 12 degrees of freedom ( 25 data points minus 13 free parameters ${ }^{3}$ ). This achieved the satisfactory fit of $\chi_{\text {d.o.f. }}^{2}=0.84$. Table 2 presents the $\chi_{\text {d.o.f. }}^{2}$ value for occultation events from 1988 to 2020 . The best-fitting parameters returned from our ray-tracing code are listed in Table 1. In particular, the reference radius pressure is about $6.72 \pm 0.48 \mu \mathrm{bar}$ ( $1 \sigma$ level error bars), which corresponds to the surface pressure of $12.36 \pm 0.38 \mu$ bar obtained from fitting the DO15 model.

In order to understand the atmospheric pressure evolution of Pluto, a numerical seasonal volatile transport model was developed by Bertrand \& Forget (2016) at the Laboratoire de Météorologie Dynamique (LMD). According to this model and observed ground-based stellar occultations (Table 2), a peak is distinguished in the pressure values. According to the results, the time-dependent atmospheric pressure of Pluto is very sensitive to the $\mathrm{N}_{2}$ ice temperature. The latter temperature is influenced by several unconstrained parameters, such as albedo and volatile distribution. Results were shown for the seasonal thermal inertia (TI) between 500 and $1500 \mathrm{~J} \mathrm{~s}^{-1 / 2} \mathrm{~m}^{-2} \mathrm{~K}^{-1}$, which is the principal parameter that can affect the $\mathrm{N}_{2}$ cycle. The stellar occultation observations showed that the pressure has been increasing since 1988 due to the maximum rate of insolation over Sputnik Planitia's glacier. So, the LMD model shows that high TI leads to more formation of seasonal $\mathrm{N}_{2}$ ice and an increase in atmospheric pressure. Over the next few years, the subsolar point will move to higher latitudes. Thus, a decrease in pressure is pre-

\footnotetext{
3 We used the same 12 free parameters as DO15 in addition to the Pluto plus Charon flux, as explained above.
}

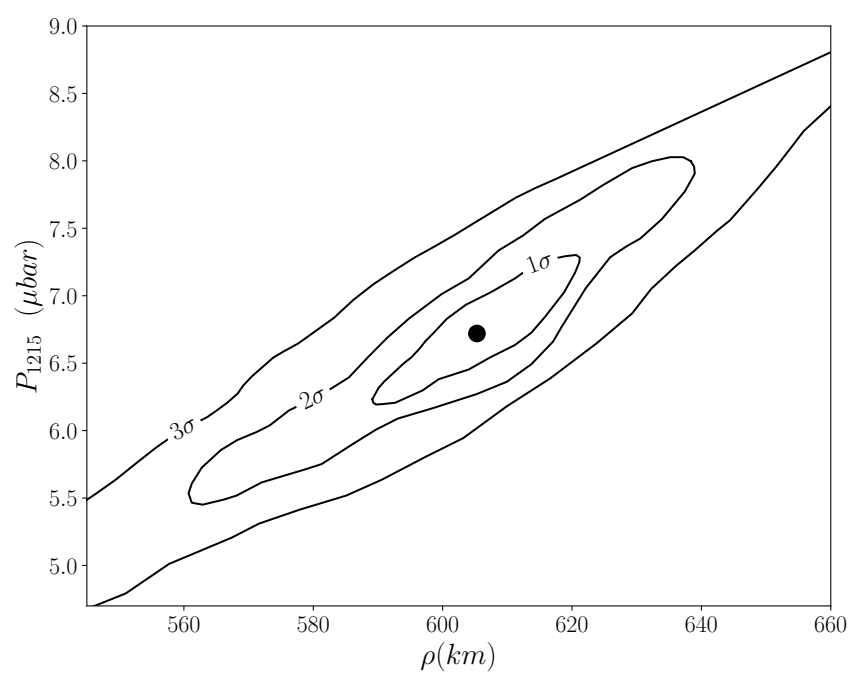

Fig. 3. $\chi^{2}$ map as a function of $P_{\text {surface }}$ and $\rho$ derived from the simultaneous fit to our light curve. The minimum $\chi^{2}$ value of 10.08 with 12 degrees of freedom is marked with a black point. The $1 \sigma\left(\chi_{\min }^{2}+1\right)$, $2 \sigma\left(\chi_{\min }^{2}+4\right)$, and $3 \sigma\left(\chi_{\min }^{2}+9\right)$ levels are also shown. The $1 \sigma$ curve provides the error bar.

dicted because of the decrease in insolation and the sublimation of $\mathrm{N}_{2}$ ice over Sputnik Planitia.

In 2019, Meza et al. (2019) constrained the LMD Pluto volatile transport model and derived a seasonal TI of $800 \mathrm{~J} \mathrm{~s}^{-1 / 2} \mathrm{~m}^{-2} \mathrm{~K}^{-1}$ and an $\mathrm{N}_{2}$ ice albedo range of $A_{\mathrm{N}_{2}}=$ $0.72-0.73$. The monotonic increase in pressure from 1988 to 2016 is consistent with this model (Meza et al. 2019).

\section{Results and conclusion}

Pluto's stellar occultation on 6 June 2020 was observed to obtain Pluto's atmospheric parameters. For the sake of finding and comparing the pressure at the specified height of the atmosphere, we satisfactorily fit our occultation light curve with the DO15 atmospheric model, taking a spherically symmetric planet and $\mathrm{N}_{2}$ pure atmosphere into account. Analysis of our single-chord photometry data demonstrates that the atmospheric pressure at the reference radius of $1215 \mathrm{~km}$ is $6.72 \pm 0.48 \mu \mathrm{bar}$.

Pluto's atmospheric pressure values from 1988 to 2016 were retrieved by Meza et al. (2019) based on the same light curve fitting model (DO15), except for the 1988 study (Table 2). Pluto's atmospheric pressure at the reference radius of $1215 \mathrm{~km}, P_{1215}$, 
Table 2. Recent atmospheric pressures of Pluto determined from ground-based stellar occultations.

\begin{tabular}{lcccc}
\hline \hline Observation & $\begin{array}{c}\text { Surface pressure } \\
(\mu \mathrm{bar})\end{array}$ & $\begin{array}{c}\text { Pressure at the radius } \\
\text { of } 1215 \mathrm{~km} \mu \mathrm{bar}\end{array}$ & $\chi_{\text {d.o.f. }}^{2}$ & Reference \\
\hline 1988 Jun. 09 & $4.28 \pm 0.44$ & $2.33 \pm 0.24$ & NA & Meza et al. (2019) \\
2002 Aug. 21 & $8.08 \pm 0.18$ & $4.42 \pm 0.09$ & 1.52 & Meza et al. (2019) \\
2007 Jun. 14 & $10.29 \pm 0.44$ & $5.6 \pm 0.24$ & 1.56 & Meza et al. (2019) \\
2008 Jun. 22 & $11.11 \pm 0.59$ & $6.05 \pm 0.32$ & 0.93 & Meza et al. (2019) \\
2008 Jun. 24 & $10.52 \pm 0.51$ & $5.73 \pm 0.21$ & 1.15 & Meza et al. (2019) \\
2010 Feb. 14 & $10.36 \pm 0.4$ & $5.64 \pm 0.22$ & 0.98 & Meza et al. (2019) \\
2010 Jun. 04 & $11.24 \pm 0.96$ & $6.12 \pm 0.52$ & 1.02 & Meza et al. (2019) \\
2011 Jun. 04 & $9.39 \pm 0.70$ & $5.11 \pm 0.38$ & 1.04 & Meza et al. (2019) \\
2012 Jul. 18 & $11.05 \pm 0.08$ & $6.07 \pm 0.04$ & 0.61 & Meza et al. (2019) \\
2013 May 04 & $12.0 \pm 0.09$ & $6.53 \pm 0.05$ & 1.20 & Meza et al. (2019) \\
2015 Jun. 29 & $12.71 \pm 0.14$ & $6.92 \pm 0.07$ & 0.84 & Meza et al. (2019) \\
2016 Jul. 19 & $12.04 \pm 0.41$ & $6.61 \pm 0.22$ & 0.86 & Meza et al. (2019) \\
2019 Jul. 17 & $9.56_{-0.34}^{+0.52}$ & $5.20_{-0.19}^{+0.28}$ & 0.84 & Arimatsu et al. (2020) \\
2020 Jun. 06 & $12.36 \pm 0.38$ & $6.72 \pm 0.48$ & 0.84 & This study \\
\hline
\end{tabular}

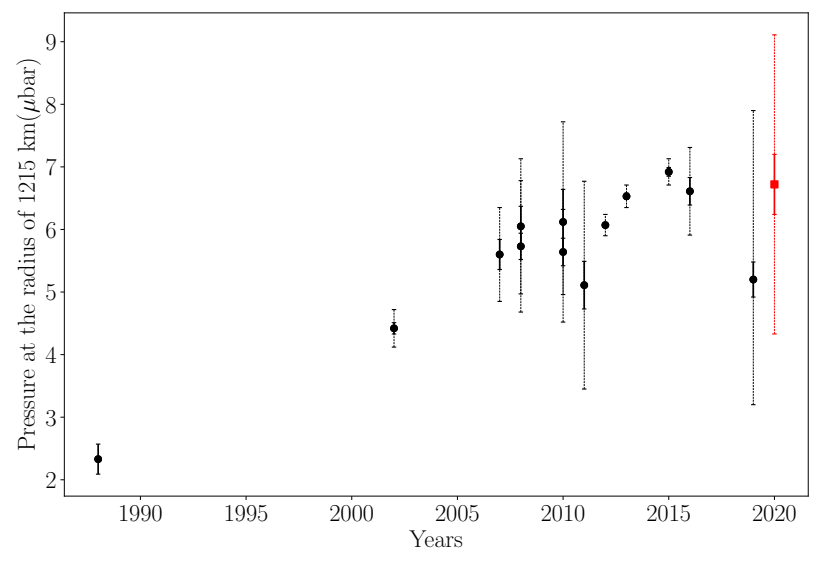

Fig. 4. Pluto's atmospheric pressure at the reference radius of $R=1215$ as a function of time. The red square is for this study. Black dots (taken from Arimatsu et al. 2020) show the other atmospheric pressures obtained from 1988 to 2019 stellar occultation measurements with their $1 \sigma$ error bars (solid lines). The $3 \sigma$ error bars are also indicated (thin lines).

is plotted as a function of time in Fig. 4 to explain our occultation result compared to previous occultation light curves from the period 1988-2019.

As shown in Fig. 4, the threefold increase in pressure observed in 2015 is consistent with the expected pressure evolution from the LMD Pluto volatile transport model (Bertrand \& Forget 2016). The pressure derived here is also consistent with the continuous increase in pressure that has been observed since 1988. Based on the results of our study, there is nominally a small, but statistically insignificant, pressure decrease of $3 \%$ from the highest reported pressure value in 2015 and an increase since 1988 by a factor of three. The latest reported stellar occultation of Pluto, by Arimatsu et al. (2020) in July 2019, showed a decrease in the pressure rate of about $21 \%$ compared to the latest reported data from a stellar occultation in 2016. The Pluto volatile transport model predicts a decrease in surface pressure over the next few years due to the orbital decline in solar insolation and more condensation of $\mathrm{N}_{2}$ ice in the colder part of Sputnik Planitia (Bertrand \& Forget 2016). However, the obtained pressure drop found by Arimatsu et al. (2020) was greater than the model predictions and is not confirmed in the present study.

This study's consistency with past studies indicates that the sublimation and condensation rate of $\mathrm{N}_{2}$ ice in Sputnik Planitia's glacier is continuing to increase because of maximum insolation over this area.

Acknowledgements. The authors thank the ERC project $n^{\circ} 669416$ "Lucky Star" for making the prediction of this event publicly available. Furthermore, we are grateful to Paul D. Maley for making editorial corrections to the text and Fatemeh Hasheminasab for preparing the Latex version of the paper. The authors would like to thank the referee and the editor for their comments and suggestions that helped to improve the paper.

\section{References}

Arimatsu, K., Hashimoto, G. L., Kagitani, M., et al. 2020, A\&A, 638, L5 Ashley, J. 2015, Mastering DeepSky Stacker (Springer), 163

Astropy Collaboration (Robitaille, T. P., et al.) 2013, A\&A, 558, A33

Bertrand, T., \& Forget, F. 2016, Nature, 540, 86

Bosh, A. S., Person, M. J., Zuluaga, C. A., et al. 2015, AAS/Division for Planetary Sciences Meeting Abstracts \#47

Collins, K. A., Kielkopf, J. F., Stassun, K. G., et al. 2017, AJ, 153, 77

Dias-Oliveira, A., Sicardy, B., Lellouch, E., et al. 2015, ApJ, 811, 53

Elliot, J. L., \& Young, L. A. 1992, AJ, 103, 991

Elliot, J. L., Dunham, E. W., Bosh, A. S., et al. 1989, Icarus, 77, 148

Hiltner, W. A. 1962, Astronomical Techniques (Chicago: University of Chicago Press)

Hubbard, W. B., Hunten, D. M., Dieters, S. W., et al. 1988, Nature, 336, 452

Meza, E., Sicardy, B., Assafin, M., et al. 2019, A\&A, 625, A42

Sicardy, B., Bolt, G., Broughton, J., et al. 2011, AJ, 141, 67

Sicardy, B., Talbot, J., Meza, E., et al. 2016, ApJ, 819, L38

Spencer, J., Grundy, W. M., Nimmo, F., et al. 2020, The Trans-Neptunian Solar System, 271

Stern, S. A., Bagenal, F., Ennico, K., et al. 2015, Science, 350, aad1815

Yelle, R. V., \& Elliot, J. L. 1997, Pluto and Charon (Part IV) (Tucson: University of Arizona Press), 347

Young, L., Sicardy, B., Widemann, T., et al. 2010, AAS/Division for Planetary Sciences Meeting Abstracts \#42 